\title{
Regulation of MTI-MMP/MMP-2/TIMP-2 axis in human placenta
}

This article was published in the following Dove Press journal:

Journal of Inflammation Research

3 October 2015

Number of times this article has been viewed

\author{
Zoë L Vincent ${ }^{1,2}$ \\ Murray D Mitchell ${ }^{3}$ \\ Anna P Ponnampalam ${ }^{1,2}$ \\ 'Liggins Institute, ${ }^{2}$ Gravida: National \\ Centre for Growth and Development, \\ University of Auckland, Auckland, \\ New Zealand; ${ }^{3}$ University of \\ Queensland Centre for Clinical \\ Research, Brisbane, QLD, Australia
}

Correspondence: Anna P Ponnampalam Liggins Institute, University of Auckland, Private Bag 32019, Auckland II42, New Zealand

Tel +649923 21II5

Fax +6493737497

Email a.ponnampalam@auckland.ac.nz
Abstract: Matrix metalloproteinases (MMPs) and specific endogenous tissue inhibitors of metalloproteinases (TIMPs) mediate rupture of the fetal membranes in both physiological and pathological conditions. MMPs and TIMPs are subject to regulation by DNA methylation in human malignancies and pre-eclampsia. To determine if membrane type 1 MMP (MT1-MMP), MMP2, and TIMP2 are regulated by DNA methylation in human placentas, we employed an in vitro model where human placental tissues were collected at term gestation and cultured with methylation inhibiting agent 5-aza-2'-deoxycytidine (AZA) and lipopolysaccharide. The results suggest that DNA methylation is not directly involved in the regulation of MT1-MMP in placental tissue; however, remodeling of chromatin by a pharmacologic agent such as AZA potentiates an infection-related increase in MT1-MMP. MT1-MMP is a powerful activator of MMP2 and this action, coupled with either no change or a decrease in TIMP2 concentrations, favors a gelatinolytic state leading to extracellular matrix degradation, which could predispose fetal membranes to rupture prematurely during inflammation.

Keywords: placenta, epigenetic regulation, DNA methylation, MMPs, labor

\section{Introduction}

Matrix metalloproteinases (MMPs) are zinc-dependent endopeptidases secreted as latent proenzymes into the extracellular space. Once activated, MMPs are capable of degrading a variety of extracellular matrix (ECM) components. ${ }^{1}$ Soluble MMPs, particularly MMP2 and MMP9, are major modulators of fetal membrane integrity throughout gestation and are responsible for membrane rupture at the onset of labor. ${ }^{2}$ Membrane type 1 MMP (MT1-MMP, also known as MMP14) is synthesized as an inactive $63 \mathrm{kDa}$ latent enzyme, which is transported to the cell membrane where it is cleaved into the active $57 \mathrm{kDa}$ form. ${ }^{3}$ In addition to degrading ECM components, MT1-MMP is a potent physiological activator of soluble MMPs. Tissue inhibitors of metalloproteinases (TIMPs) are endogenous inhibitors of MMPs and regulate their activation by binding in a 1:1 stoichiometry; ${ }^{4}$ shifting the balance between TIMPs and active MMPs results in excessive proteolytic activity. ${ }^{5}$

Latent MMP2 (pro-MMP2) is secreted as a $72 \mathrm{kDa}$ proenzyme, and is not readily activated into its active $68 \mathrm{kDa}$ form by general proteolytic cleavage. ${ }^{6-10}$ Instead, activation is mediated at the cell surface via a series of reactions involving MT1-MMP and TIMP2. ${ }^{4}$ A pro-MMP2/TIMP2 complex is formed via the C-terminal of both proteins, leaving the inhibitory N-terminal of TIMP2 free to bind MT1-MMP at the cell surface. Once at the cell surface, the pro-MMP2 is activated by a second MT1-MMP, 
which is free of TIMPs. Alternatively, if TIMP2 is already inhibiting MT1-MMP, this complex may act as a receptor for pro-MMP2. ${ }^{4,5,11}$

The regulation of MT1-MMP in human placenta is not well understood. MT1-MMP has previously been detected in fetal membranes collected at term gestation from nonlaboring cesarean section (CS) deliveries, and from normal vaginal deliveries with the spontaneous onset of labor (spontaneous vaginal delivery [SVD]). Fortunato et al have reported that MT1-MMP mRNA and protein were present in the fetal membranes and that no differences were detected following culture with lipopolysaccharide (LPS), suggesting constitutive expression of this MMP in the fetal membranes. $^{12}$

There is conflicting information about the role of MMP2 in fetal membrane rupture at term gestation. MMP2 has been reported to be constitutively expressed in fetal membranes throughout gestation. ${ }^{13,14}$ However, it has more recently been shown to increase with gestational age in amnion, with significantly increased levels at term labor. ${ }^{15} \mathrm{MMP} 2$ has also been implicated in preterm premature rupture of the fetal membranes ( $\mathrm{pPROM})^{16}$ and is increased in the presence of LPS. ${ }^{17}$ TIMP2 protein increases with advancing gestational age, and decreases with preterm and term labor, pPROM, and in the presence of intra-amniotic infection. ${ }^{18}$ Furthermore, TIMP2 protein is decreased in cultured fetal membranes exposed to LPS. $^{17,18}$

Epigenetic regulation refers to modifications to DNA and chromatin that result in heritable changes in gene expression independent of the genetic sequence and include DNA methylation and histone modifications. ${ }^{19}$ DNA methylation refers to the addition of a methyl group to the cytosine ring in DNA in the context of a $\mathrm{CpG}$ dinucleotide, to form methyl cytosine (5-mC). The silencing of TIMP genes via promoter methylation is a hallmark of cancer development, and the MT1-MMP/ MMP2/TIMP2 axis is regulated by a combination of DNA methylation and histone modifications in cancer cells. ${ }^{20}$

In the current study, we hypothesize that MT1-MMP, MMP2, and TIMP2 are regulated by DNA methylation in human term placenta and that aberrant methylation leading to altered gene expression may contribute to fetal membrane rupture.

\section{Materials and methods}

\section{Tissue collection and explant system}

The present study was designed in accordance with the principles set out in the Declaration of Helsinki. Ethical approval for the study was obtained from Northern X Regional
Ethics Committee (NTX/10/07/062), and tissues were collected following written informed consent. Villous placental tissues were collected from women at term with uncomplicated, singleton pregnancies (38-40 weeks gestation) following elective CS (mean maternal age 35 years, mean gestational age 38 weeks). Tissue culture protocol including doses and length of treatments were based on a previously published in vitro tissue explant system, ${ }^{21-23}$ with modifications.

Samples of villous tissue were taken randomly across the placenta from midsections of cotyledons (halfway between the maternal and fetal sides). Large vessels were removed using blunt dissection and leaving only villous tissue, which was further dissected into $20 \mathrm{mg}$ pieces. Villous explants were plated separately (six pieces per well) and were equilibrated for 24 hours in Dulbecco's Modified Eagle's Medium (DMEM)/F12 containing L-glutamate (Thermo Fisher Scientific, Waltham, MA, USA) with 10\% fetal bovine serum (FBS) (Thermo Fisher Scientific) and 1\% antibiotic solution (final concentrations $100 \mathrm{U} / \mathrm{mL}$ penicillin and $100 \mu \mathrm{g}$ streptomycin; Thermo Fisher Scientific) in a humidified atmosphere of $5 \% \mathrm{CO}_{2}$ and $8 \% \mathrm{O}_{2}$.

After equilibration, tissues were washed and media were replaced with DMEM/F12 supplemented with $0.1 \%$ bovine gamma globulin (Sigma-Aldrich Co, St Louis, MO, USA) containing $5 \mu \mathrm{M}$ 5-aza-2'-deoxycytidine (AZA; SigmaAldrich Co) or dimethyl sulfoxide (DMSO, a vehicle control; Sigma-Aldrich Co). Following 48 hours culture, tissues were extensively washed in sterile phosphate-buffered saline (PBS), and tissues were further incubated in the presence or absence of $5 \mu \mathrm{g} / \mathrm{mL}$ LPS and Escherichia coli 055.B5 (Sigma-Aldrich Co). Tissues were cultured with LPS to determine if an inflammatory response induced changes in TIMP1 expression and/or DNA methylation. Culture was terminated at 24 hours and 48 hours post-LPS treatment; tissues were snap-frozen, and conditioned media were reserved. Tissues and media samples were stored at $-80^{\circ} \mathrm{C}$ and $-20^{\circ} \mathrm{C}$, respectively.

\section{RNA extraction and quantitative real-time polymerase chain reaction}

Total RNA was isolated from tissues using Trizol (Thermo Fisher Scientific) according to the manufacturer's instructions. RNA concentrations were quantified using a NanoDrop ND-1000 spectrophotometer (Thermo Fisher Scientific). Following DNase treatment, reverse transcription and cDNA synthesis were performed using a Transcriptor First Strand cDNA Synthesis Kit (Hoffman-La Roche Ltd, Basel, Switzerland) according to the manufacturer's 
instructions using $1 \mu \mathrm{g}$ of total RNA for each preparation. The resulting cDNA was stored at $-20^{\circ} \mathrm{C}$ until required.

MT1-MMP, MMP2, and TIMP2 mRNA expression was analyzed by quantitative real-time polymerase chain reaction (qRT-PCR) using the LightCycler ${ }^{\circledR} 480$ and LightCycler $480 \mathrm{SYBR}^{\circledR}$ Green Master Mix (Hoffman-La Roche Ltd). Gene-specific primers used are detailed in Table 1. RPLPO and $R P L 13 a$ were used as endogenous controls to normalize gene expression, and the average transcript quantity in treated tissue explants was calculated using the delta-delta CT method. ${ }^{24}$

\section{Western blotting}

Western blotting was performed on whole cell lysates using rabbit monoclonal anti-MT1-MMP antibody (1:1,000 dilution, product number ab51074, Abcam, Cambridge, UK). Proteins were separated by weight by sodium dodecyl sulfate polyacrylamide gel electrophoresis using 4\%-12\% Bis-Tris gels (Thermo Fisher Scientific), and were transferred onto polyvinylidene fluoride membrane. Following pre-incubation with a blocking solution, membranes were incubated in primary antibody overnight. The membranes were then extensively washed, incubated in horseradish peroxidase (HRP)-conjugated secondary antibody (1:10,000 dilution, product number A5045; Sigma Aldrich Co), and were visualized using Pierce Super-

Table I Gene-specific primers used for qRT-PCR

\begin{tabular}{|c|c|c|c|}
\hline Gene & Primer se & quence & Amplicon \\
\hline MMP2 & Sense & 5'-ACA GTG CAT CTC AGC & 323 \\
\hline & & CCA CAT AGT-3' & \\
\hline & Antisense & 5'-AAG CTC TGA CCT TTC & \\
\hline & & CAG CAG ACA-3' & \\
\hline MTI-MMP & Sense & 5'- ATC ATG GCA CCC TTT & 253 \\
\hline & & TAC CA-3' & \\
\hline & Antisense & 5'-AGC GCT CCT TGA AGA & \\
\hline & & CAA AC-3' & \\
\hline TIMP2 & Sense & 5'-CCC TCC TCG GCA GTG & 154 \\
\hline & & TGT-3' & \\
\hline & Antisense & 5'-GGC TCT TCT TCT GGG & \\
\hline & & TGG TG-3' & \\
\hline RPLPO & Sense & 5'-AGA AAC TGC TGC CTC & 223 \\
\hline & & ATA TCC G-3' & \\
\hline & Antisense & $5^{\prime}$-CCC CTG GAG ATT TTA & \\
\hline & & GTG GTG A-3' & \\
\hline RPLI $3 a$ & Sense & $5^{\prime}$-GCC CTA CGA CAA GAA & 117 \\
\hline & & AAA GCG-3' & \\
\hline & Antisense & 5'-TAC TTC CAG CCA ACC & \\
\hline & & TCG TGA-3' & \\
\hline
\end{tabular}

Abbreviations: qRT-PCT, quantitative real-time polymerase chain reaction; $M M P 2$, matrix metalloproteinase type 2; MTI-MMP, membrane type I matrix metalloproteinase; TIMP2, tissue inhibitor of metalloproteinase type 2; RPLPO, large ribosomal protein; $R P L 13$, ribosomal protein type 13; bp, base pairs.
Signal ${ }^{\circledR}$ West Dura Extended Duration Substrate (Thermo Fisher Scientific). Relative protein levels were obtained using densitometric quantification within the linear range (Quantity One $^{\circledR}$ software; Bio-Rad Laboratories Inc., Hercules, CA, USA) and were normalized to $\beta$-actin.

\section{Gelatin zymography}

MMP2 activity was measured in conditioned media from placental explant cultures using gelatin zymography. Samples were separated by electrophoresis ( $125 \mathrm{~V}$ for 90 minutes) on Novex ${ }^{\circledR} 10 \%$ gelatin zymogram gels (Thermo Fisher Scientific). Gels were incubated in denaturing buffer for 30 minutes, and then in developing buffer overnight at $37^{\circ} \mathrm{C}$. Gels were stained in a solution containing $0.3 \%$ coomassie blue, after which areas on the gel that had been degraded appeared as clear bands against a dark background. Molecular weights were determined using a standard of conditioned media from HT-1080 human fibrosarcoma cells, which constitutively express MMP2 and MMP9. Gels were scanned with a GS-800 calibrated densitometer (Bio-Rad Laboratories Inc.) and were analyzed using Quantity One software (Bio-Rad Laboratories Inc.).

\section{Enzyme-linked immunosorbent assay}

Total TIMP2 protein in conditioned media from tissue explant experiments was measured using a human TIMP2 DuoSet $^{\circledR}$ enzyme-linked immunosorbent assay (ELISA) kit (R\&D Systems Inc., Minneapolis, MN, USA) according to the manufacturer's instructions. Optical density was read at $450 \mathrm{~nm}$ and was corrected with $540 \mathrm{~nm}$ on a Synergy ${ }^{\mathrm{TM}}$ 2 multimode plate reader (BioTek, Winooski, VT, USA). Levels of secreted TIMP2 were normalized with respect to the total protein content as measured by microplate bicinchoninic assay protein assay kit (Thermo Fisher Scientific).

\section{DNA extraction and methylation analysis of MTI-MMP promoter}

Genomic DNA was extracted from tissues using the QiaAMP DNA extraction kit (Qiagen NV, Venlo, the Netherlands) as per manufacturer's instructions and quantified using a NanoDrop ND-1000 spectrophotometer.

Methylation analysis using the Sequenom ${ }^{\mathrm{TM}}$ EpiTyper $^{\mathrm{B}}$ MassARRAY platform was undertaken by the Australian Genome Research Facility (AGRF). Primer sets to cover two regions ( $\mathrm{A}$ and $\mathrm{B}$ ) of the major $\mathrm{CpG}$ island in the MT1-MMP promoter region were designed in-house by AGRF. Region A covered $30 \mathrm{CpG}$ sites and region $\mathrm{B}$ covered $38 \mathrm{CpG}$ sites. Primers used for Sequenom analysis are detailed in Table 2. 
Table 2 Sequenom ${ }^{\mathrm{TM}}$ primers used for MTI-MMP promoter methylation analysis

\begin{tabular}{llllll}
\hline & Primer & Start site & Size & GC $\%$ & Sequence \\
\hline Region A & Left & 71 & 30 & 46.67 & GGAAAAGAGGAGAAGAGTAAATAGGTATTT \\
& Right & 450 & 27 & 59.26 & AATAAAAACTATCCCCTAAAAAACCCC \\
& Product size: 380, Tm: 72.0, CpGs in product: 30 & & \\
Region B & Left & 377 & 25 & 62.50 & GGGTTTTGTTTTTAGTTTTGGAAT \\
& Right & I,036 & 25 & 50.00 & ACTCACAAAAAAAACTTTATATATAC \\
& Product size: 360, Tm: 72.1, CpGs in product: 38 & &
\end{tabular}

Abbreviations: MTI-MMP, membrane type I matrix metalloproteinase; GC\%, guanine-cytosine percentage; Tm, melting temperature; CpGs, dense region of cytosine and guanine nucleotides.

Methylation analysis was carried out on bisulfite-converted DNA using $200 \mathrm{ng}$ for each preparation. Methylation levels for each $\mathrm{CpG}$ unit were expressed as a percentage, which was calculated from the ratio of mass signals between methylated and nonmethylated DNA in each sample.

\section{Chromatin accessibility assay}

Chromatin assembly of treated villous explant tissues was carried out using the EpiQuik chromatin accessibility assay kit (Epigentek, Farmingdale, NY, USA). Chromatin was isolated from tissues and treated with a nuclease (Nse) mix. DNA was then isolated and amplified using quantitative PCR and gene-specific primers for MT1-MMP (Table 1). Control primers were provided to determine the successful digestion of the chromatin. The fold enrichment (FE) was calculated by the ratio of amplification efficiency of the Nse-treated DNA sample over that of the control sample not treated with nuclease (No-Nse):

$$
\left(\mathrm{FE}=2^{\text {(Nse CT - no-Nse CT) }} \times 100 \%\right)
$$

Changes in chromatin structure in treated tissues were identified by the degree of $\mathrm{Ct}$ shift between digested and undigested samples. DNA in heterochromatin was inaccessible to the nucleases, resulting in insignificant $\mathrm{Ct}$ shifts between digested and undigested (control) samples, whereas DNA in euchromatin was accessible to nucleases, resulting in a large $\mathrm{Ct}$ shift.

\section{Statistical analysis}

Statistical analyses were performed using GraphPad Prism version 6.0 for Windows (GraphPad Software Inc., La Jolla, CA, USA). Comparisons between tissues collected from CS and SVD pregnancies were carried out using the Mann-Whitney $U$ test. One-way analysis of variance was used for explant experiments and if significantly different ( $\alpha=0.05$ ), post hoc multiple comparisons using Dunn's test were performed for comparison to time-matched controls. Values are presented as mean \pm standard error of the mean (SEM).

\section{Results}

\section{Effect of AZA and LPS treatments on MTI-MMP mRNA and protein}

$M T 1-M M P$ mRNA was significantly increased in placental explants pretreated with AZA and then stimulated with LPS for 24 hours or 48 hours compared to controls. Significant increase was also observed in explants following LPS stimulation alone for 48 hours (Figure 1A). MT1-MMP protein was significantly increased in placental explants pre-treated with AZA and then stimulated with LPS for 24 hours and 48 hours, and treated with LPS alone for 24 hours (Figure 1B).

\section{Effect of AZA and LPS treatments on MMP2 $m R N A$ and protein secretion}

$M M P 2$ mRNA expression was not altered by AZA or LPS treatment alone or in combination in any tissue (data not shown). MMP2 activity, however, was significantly increased in the culture media from placental explants pre-treated with AZA and subsequently cultured with LPS for 48 hours (Figure 2).

\section{Effect of AZA and LPS treatments on TIMP2 mRNA and protein secretion}

There were no significant differences in TIMP2 mRNA expression in relation to any treatment in any tissue (data not shown). TIMP2 protein production was significantly decreased in the culture media from placental explants pre-treated with AZA and subsequently cultured with LPS for 48 hours (Figure 3).

\section{Methylation analysis of MTI-MMP promoter region}

MT1-MMP promoter was generally hypomethylated across all tissue explants (ranging $4 \%-6 \%$ on average). In addition, no changes in methylation were observed in cultured tissue explants in response to any treatment (Figure 4). 
A

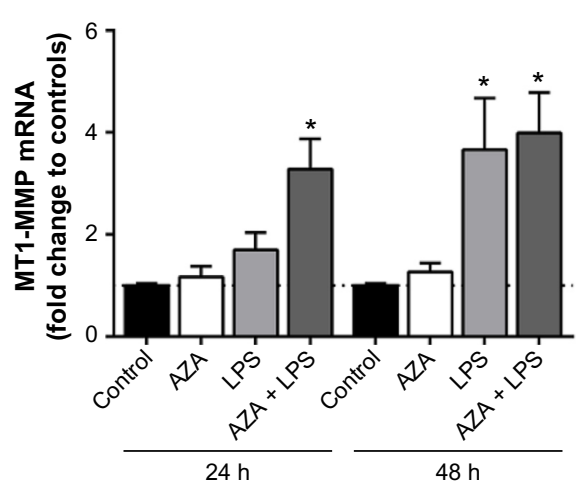

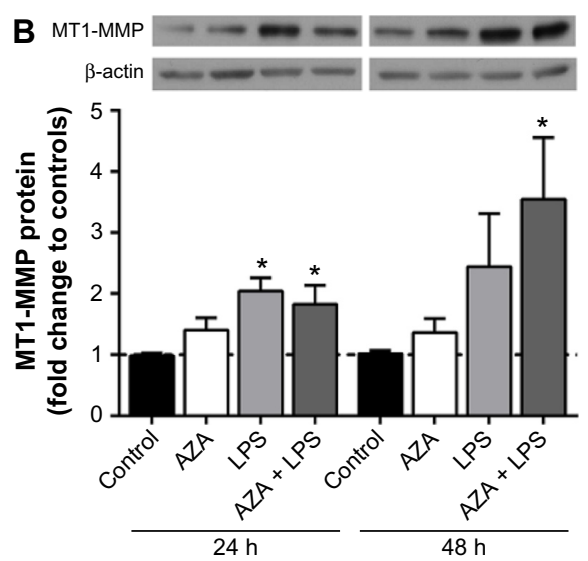

Figure I MTI-MMP mRNA and protein expression in villous explants.

Notes: (A) qRT-PCR was used to detect MTI-MMP mRNA expression in tissues treated with/without $5 \mu \mathrm{g} / \mathrm{mL}$ LPS with/without prior $5 \mu$ M AZA treatment. MTI-MMP mRNA was significantly increased in explants pre-treated with AZA and then cultured with LPS for 24 hours and 48 hours. LPS treatment alone increased MTI-MMP mRNA in explants after 48 hours. (B) Western blotting was used to detect MTI-MMP protein in tissues treated with/without $5 \mu$ g/mL LPS with/without prior $5 \mu$ M AZA treatment. MTI-MMP protein was significantly increased in placental explants pre-treated with AZA and then stimulated with LPS for 24 hours and 48 hours and in MTI-MMP protein treated with LPS alone for 24 hours. In (A), data are presented as mean fold change in MTI-MMP transcript normalized to RPLPO and RPL / $3 a$ compared to time-matched controls ( \pm SEM; $n=8$ in each group), ${ }^{*} P<0.05$. In $(B)$, data are presented as mean fold change in MTI-MMP protein normalized to $\beta$-actin optical density, compared to timematched controls $( \pm$ SEM; $n=8$ in each group); $* P<0.05$.

Abbreviations: qRT-PCR, quantitative real-time polymerase chain reaction; MTI-MMP, membrane type I matrix metalloproteinase; LPS, lipopolysaccharide; AZA, 5-aza2'-deoxycytidine; RPLPO, large ribosomal protein; RPL I3, ribosomal protein type 13; SEM, standard error of the mean; $h$, hours; $\mathrm{n}$, number in each group.

\section{Chromatin accessibility of treated tissue explants}

Changes in chromatin structure were most evident in villous tissue explants treated with AZA and subsequently exposed to LPS, as shown by the large Ct shift between digested and undigested samples (Figure 5). Due to sample constraints,

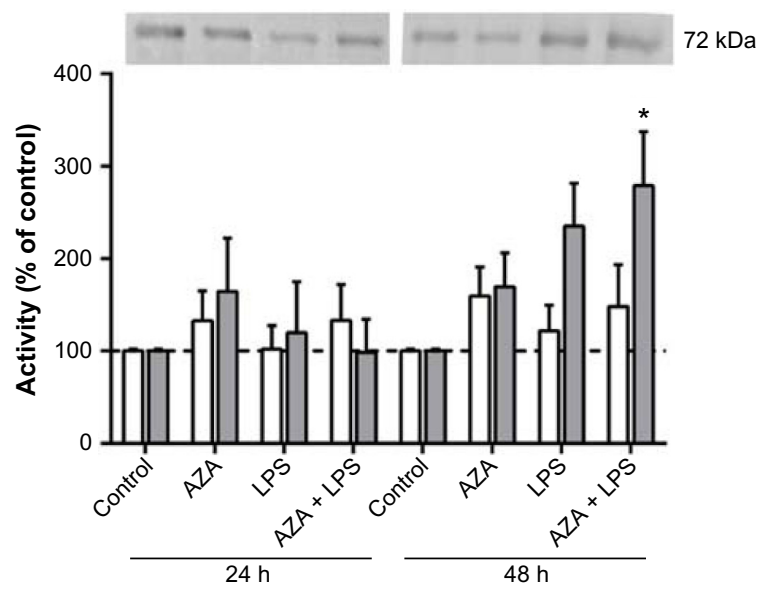

Figure 2 Secreted MMP2 activity from villous explants.

Notes: Gelatin zymography was used to assess MMP2 activity in conditioned media from tissue explants treated with/without $5 \mu \mathrm{g} / \mathrm{mL}$ LPS with/without prior $5 \mu \mathrm{M}$ AZA treatment. Pro-MMP2 activity is shown by white bars; activated MMP2 activity is shown by gray bars. A representative zymogram is shown above the graph. Levels of the secreted MMPs were normalized with respect to the total protein content and expressed as a percentage of time-matched controls (mean \pm SEM; $n=8$ in each group); $* P<0.05$.

Abbreviations: MMPs, matrix metalloproteinases; MMP2, MMP type 2; pro-MMP2, latent MMP2; LPS, lipopolysaccharide; AZA, 5-aza-2'-deoxycytidine; SEM, standard error of the mean; $h$, hours; $n$, number in each group. chromatin accessibility was only assessed in two villous sample sets, resulting in large variation. While no significant differences were noted, a relative increase was seen in AZA + LPS treatment groups.

\section{Discussion}

The present study investigated the response of MT1-MMP, MMP2, and TIMP2 to methylation inhibiting (AZA) and immunomodulating (LPS) treatments in term human gestational tissue explants. We report an increase in MT1-MMP mRNA and protein in response to AZA treatment followed by LPS stimulation. In addition, we examined promoter-specific methylation of the $M T 1-M M P$ gene.

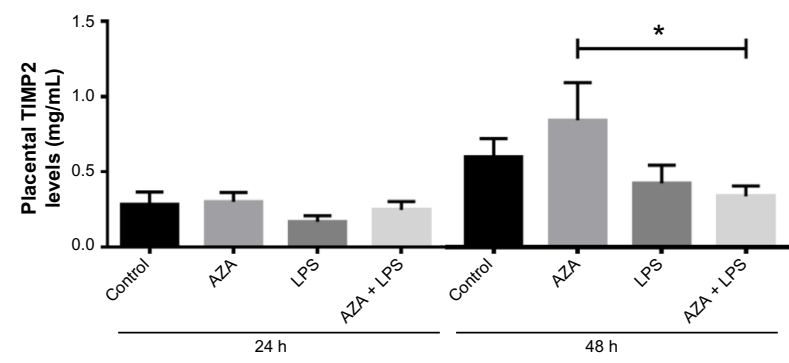

Figure 3 Secreted TIMP2 protein from villous explants.

Notes: ELISA was used to assess TIMP2 protein in conditioned media from tissue explants treated with/without $5 \mu \mathrm{g} / \mathrm{mL}$ LPS with/without prior $5 \mu \mathrm{M}$ AZA treatment. Levels of secreted TIMP2 were normalized with respect to the total protein content (mean \pm SEM; $n=8$ in each group); $* P<0.05$.

Abbreviations: TIMP2, tissue inhibitor of metalloproteinase type 2; ELISA, enzymelinked immunosorbent assay; LPS, lipopolysaccharide; AZA, 5-aza-2'-deoxycytidine; SEM, standard error of the mean; h, hours; $n$, number in each group. 


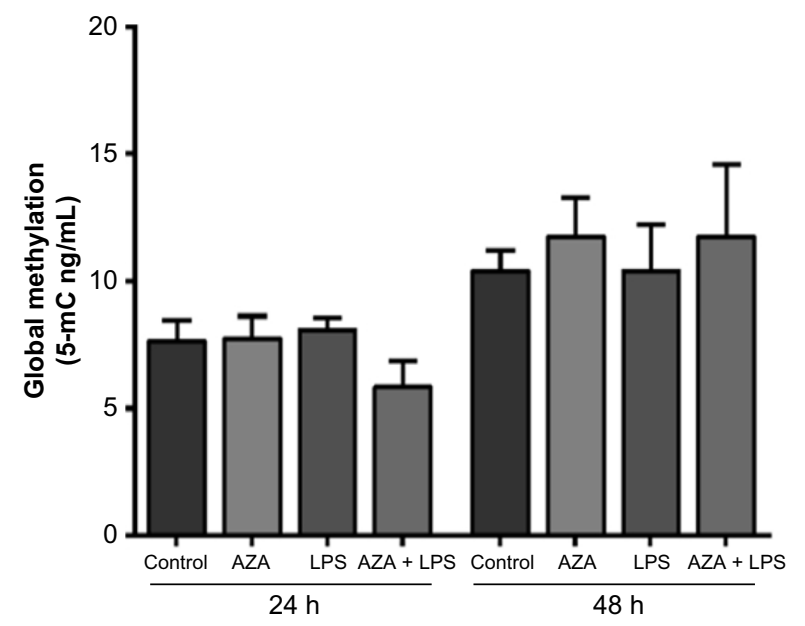

Figure $4 \mathrm{MTI}-\mathrm{MMP}$ promoter methylation in villous explants.

Notes: Sequenom ${ }^{\mathrm{TM}}$ EpiTyper $^{\circledR}$ MassARRAY was used to measure methylated cytosine residues $(5-\mathrm{mC})$ within the $\mathrm{MTI}$-MMP promoter in tissues treated with $5 \mu \mathrm{g} /$ $\mathrm{mL}$ LPS with/without prior $5 \mu$ MAZA treatment. \% of 5-Methylcytosine (\%5-mC) was calculated from the ratio of mass signals between methylated and nonmethylated DNA in each sample. Data are presented as mean \%5-mC across all CpGs \pm SEM ( $n=8)$. Abbreviations: MTI-MMP, membrane type I matrix metalloproteinase; LPS, lipopolysaccharide; AZA, 5-aza-2'-deoxycytidine; SEM, standard error of the mean; $h$, hours; $n$, number in each group.

A previous study reported that $M T 1-M M P$ mRNA was not increased by LPS treatment in amnion-chorion fetal membrane samples. ${ }^{12}$ We observed an increase in MT1MMP transcription and protein treatment with LPS alone for 24 hours and 48 hours, respectively. When AZA and LPS treatments were combined, we observed a significant increase in MT1-MMP mRNA at 24 hours and 48 hours, and in MT1-MMP protein at 48 hours.

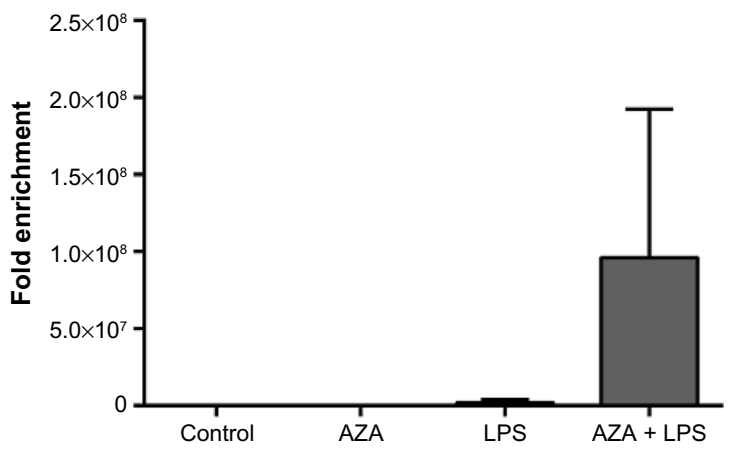

Figure 5 Chromatin accessibility assay of treated villous explants.

Notes: Chromatin structures of treated villous explants were determined by specific nucleases. DNA in heterochromatin was inaccessible to the nucleases, resulting in insignificant $\mathrm{Ct}$ shifts between digested and undigested samples, whereas DNA in euchromatin was accessible to nucleases, resulting in a large $\mathrm{Ct}$ shift shown as fold enrichment. Treatment with combined AZA and LPS resulted in large fold enrichment, signifying the DNA is more open than in the control, AZA alone, or LPS alone treatments. Data are presented as the ratio of amplification efficiency of the Nse-treated DNA sample over that of the No-Nse (control) sample (mean fold enrichment) $\pm \operatorname{SEM}(n=2)$.

Abbreviations: LPS, lipopolysaccharide; AZA, 5-aza-2'-deoxycytidine; Nse, nuclease; SEM, standard error of the mean; $n$, number in each group; No-Nse, control samples not treated with nuclease.
TIMP2 protein was decreased in tissues treated with AZA and subsequently stimulated with LPS. Furthermore, although not significant, there is an apparent decrease in TIMP2 protein secretion in tissues treated with LPS only at both time points. This observation is supported by previous studies, which reported a decrease in TIMP2 protein secretion by gestational membranes treated with LPS. ${ }^{25,26}$ Furthermore, decreased TIMP 2 expression has previously been correlated to promoter hypermethylation. ${ }^{27}$

It was interesting to note that although AZA treatment had no effect basally, when combined with LPS, the two treatments appeared to act synergistically to increase MT1-MMP mRNA and protein production. AZA is a methylation-inhibiting agent that is incorporated into the DNA as a cytidine analog and prevents methylation by irreversibly binding to DNA methyltransferases, leading to passive hypomethylation over successive rounds of cell division. ${ }^{28}$ Interrogation of the $M T 1-M M P$ promoter revealed hypomethylation in all term gestational tissues. Furthermore, no change in $M T 1-M M P$ promoter-specific methylation was observed in explant tissues in response to culture treatments. Thus, as $M T 1-M M P$ was hypomethylated in the placenta, any AZA-induced changes in methylation were negligible.

It is becoming increasingly apparent that AZA has more far-reaching effects than hypomethylation alone; evidence suggests that AZA is capable of inducing reorganization of the chromatin structure. ${ }^{29,30}$ In this way, AZA may be enhancing the availability of transcriptional regulatory elements in the $M T 1-M M P$ promoter, or directly interacting with the transcription factors themselves. Preliminary analysis of the chromatin structure of treated explants was assessed using a commercially available kit, which determines the extent of chromatin accessibility to exogenous nucleases. We observed that, for this study, villous explants treated with AZA and LPS had a more open chromatin structure compared to controls, AZA treatments, or LPS treatments alone. This result was represented by a larger shift in qRT- PCR Ct values, calculated as fold enrichment between digested and undigested samples.

A major limitation of this experiment was the low sample number, due to sample constraints. Although significant differences were not noted, the apparent increase observed between treatment groups would be clarified by an increase in sample number. AZA has been shown to reactivate gene expression independent of DNA methylation. ${ }^{31,32}$ AZA can act as an inhibitor of histone methylation, which may account for the reexpression of unmethylated genes. ${ }^{33}$ Both hypomethylation of $\mathrm{CpG}$ islands and low levels of histone 
H3 lysine-27 trimethylation are features of transcriptionally active $M T 1-M M P$ in cancer cell lines. ${ }^{34}$

Co-treatment of human colorectal cancer cell lines with combined AZA and pro-inflammatory cytokines IL-1, IL-6, or TNF- $\alpha$ induces $M M P 2$ and $M M P 9$ mRNA to a greater extent than cytokine treatment alone. This cooperative regulation is due to functional interactions between AZA and transcription factors c-Jun and C/EBP. ${ }^{35} M T 1-M M P$ contains a number of regulatory binding sites in its promoter, including several for C/EBP- $\beta$; thus, the direct interaction between AZA and transcription factors is a potential mechanism of $M T 1-M M P$ mRNA regulation in the placenta and fetal membranes in the presence of inflammation. ${ }^{36}$ Although we did not observe increased MMP 2 mRNA with combined AZA and LPS treatments, MT1-MMP induction resulted in a significant increase in MMP2 activity in placenta explants after 48 hours.

AZA could also be increasing the expression of toll-like receptors (TLRs) in these tissues, leading to enhanced LPS stimulation of MT1-MMP. TLRs are innate immune receptors that are the principle sensors of bacterial pathogens, and are highly expressed by gestational tissues. ${ }^{37}$ TLR type 4 (TLR4) mediates LPS-induced immune responses, and is methylated in human intestinal epithelial cell lines and mice embryonic stem cells. ${ }^{38,39}$ Treatment with AZA restores TLR4 expression in these cells, thereby enhancing LPS responsiveness. In one study, TLR4 reactivation was augmented by combined AZA and TSA (a histone deacetylase inhibitor) treatments, suggesting cooperative control of TLR4 by DNA methylation and histone modifications. ${ }^{38}$

pPROM is associated with increased MMP2 and MT1MMP concentrations, and decreased TIMP2 protein in fetal membranes and amniotic fluid. ${ }^{13,16,18}$ In the current study, we report significantly increased MT1-MMP mRNA and protein and MMP2 activity with an associated decrease in TIMP2 protein in placental explants treated with combined AZA and LPS treatments. MT1-MMP and TIMP2 are major mediators of MMP2 activity, and while high concentrations of MT1-MMP facilitate the activation of MMP2, low TIMP2 concentration would result in less inhibition of the activated enzymes. ${ }^{40}$ Increased MMP levels in the presence of unchanged or decreased TIMP2 would shift the balance in favour of gelatinase activity, resulting in ECM degradation.

\section{Conclusion}

Our results imply that promoter methylation is not directly involved in the regulation of MT1-MMP in human placenta. Rather, alterations in chromatin structure instigated by pharmacologic agents such as AZA could expose regulatory regions in the $M T 1-M M P$ promoter, allowing for the binding of transcription factors. Furthermore, recent evidence demonstrates the direct interaction between AZA and transcriptional factors in the presence of inflammatory mediators leads to increased $M M P$ transcription. Together, this evidence supports a mechanistic link between epigenetic modifications and inflammation in the control of MMP expression. Enhanced MT1-MMP in gestational tissues alongside an associated increase in activation of MMP2 would alter the balance of MMPs and TIMPs, favouring a proteolytic state and thereby making the fetal membranes vulnerable to ECM degradation and subsequent membrane rupture.

\section{Acknowledgments}

We thank staff and patients at Auckland City Hospital for donation and collection of tissue samples. We also thank Dr Devaki De Silva for technical input.

\section{Disclosure}

The authors report no conflicts of interest in this work.

\section{References}

1. Reich R, Thompson EW, Iwamoto Y, et al. Effects of inhibitors of plasminogen activator, serine proteinases, and collagenase IV on the invasion of basement membranes by metastatic cells. Cancer Res. 1988; 48(12):3307-3312.

2. Weiss A, Goldman S, Shalev E. The matrix metalloproteinases (MMPS) in the decidua and fetal membranes. Front Biosci. 2007;12:649-659.

3. Nagase H, Woessner JF Jr. Matrix metalloproteinases. J Biol Chem. 1999;274(31):21491-21494.

4. Visse R, Nagase H. Matrix metalloproteinases and tissue inhibitors of metalloproteinases: structure, function, and biochemistry. Circ Res. 2003;92(8):827-839.

5. Nagase H, Visse R, Murphy G. Structure and function of matrix metalloproteinases and TIMPs. Cardiovasc Res. 2006;69(3):562-573.

6. Woessner JF Jr. Matrix metalloproteinases and their inhibitors in connective tissue remodeling. FASEB J. 1991;5(8):2145-2154.

7. Murphy G, Cockett MI, Ward RV, Docherty AJ. Matrix metalloproteinase degradation of elastin, type IV collagen and proteoglycan. A quantitative comparison of the activities of $95 \mathrm{kDa}$ and $72 \mathrm{kDa}$ gelatinases, stromelysins-1 and -2 and punctuated metalloproteinase (PUMP). Biochem J. 1991;277(Pt 1):277-279.

8. Murphy G, Ward R, Hembry RM, Reynolds JJ, Kuhn K, Tryggvason K. Characterization of gelatinase from pig polymorphonuclear leucocytes. A metalloproteinase resembling tumour type IV collagenase. Biochem J. 1989;258(2):463-472.

9. Hipps DS, Hembry RM, Docherty AJ, Reynolds JJ, Murphy G. Purification and characterization of human $72-\mathrm{kDa}$ gelatinase (type IV collagenase). Use of immunolocalisation to demonstrate the non-coordinate regulation of the $72-\mathrm{kDa}$ and $95-\mathrm{kDa}$ gelatinases by human fibroblasts. Biol Chem Hoppe Seyler. 1991;372(4):287-296.

10. Woessner JF Jr. The family of matrix metalloproteinases. Ann NY Acad Sci. 1994;732:11-21.

11. Nelson AR, Fingleton B, Rothenberg ML, Matrisian LM. Matrix metalloproteinases: biologic activity and clinical implications. $J$ Clin Oncol. 2000;18(5):1135-1149.

12. Fortunato SJ, Menon R, Lombardi SJ. Expression of a progelatinase activator (MT1-MMP) in human fetal membranes. Am J Reprod Immunol. 1998;39(5):316-322. 
13. Athayde N, Romero R, Gomez R, et al. Matrix metalloproteinases-9 in preterm and term human parturition. J Matern Fetal Med. 1999;8(5): 213-219.

14. Xu P, Alfaidy N, Challis JR. Expression of matrix metalloproteinase (MMP)-2 and MMP-9 in human placenta and fetal membranes in relation to preterm and term labor. J Clin Endocrinol Metab. 2002;87(3): 1353-1361.

15. Ota A, Yonemoto H, Someya A, Itoh S, Kinoshita K, Nagaoka I. Changes in matrix metalloproteinase 2 activities in amniochorions during premature rupture of membranes. J Soc Gynecol Investig. 2006;13(8):592-597.

16. Chelbi ST, Mondon F, Jammes H, et al. Expressional and epigenetic alterations of placental serine protease inhibitors: SERPINA3 is a potential marker of preeclampsia. Hypertension. 2007;49(1):76-83.

17. Fortunato SJ, Menon R, Lombardi SJ. MMP/TIMP imbalance in amniotic fluid during PROM: an indirect support for endogenous pathway to membrane rupture. J Perinat Med. 1999;27(5):362-368.

18. Maymon E, Romero R, Pacora P, et al. A role for the $72 \mathrm{kDa}$ gelatinase (MMP-2) and its inhibitor (TIMP-2) in human parturition, premature rupture of membranes and intraamniotic infection. J Perinat Med. 2001; 29(4):308-316.

19. Probst AV, Dunleavy E, Almouzni G. Epigenetic inheritance during the cell cycle. Nat Rev Mol Cell Biol. 2009;10(3):192-206.

20. Chernov AV, Sounni NE, Remacle AG, Strongin AY. Epigenetic control of the invasion-promoting MT1-MMP/MMP-2/TIMP-2 axis in cancer cells. J Biol Chem. 2009;284(19):12727-12734.

21. Mitchell MD. Unique suppression of prostaglandin $H$ synthase-2 expression by inhibition of histone deacetylation, specifically in human amnion but not adjacent choriodecidua. Mol Biol Cell. 2006;17(1):549-553.

22. Sato TA, Mitchell MD. Molecular inhibition of histone deacetylation results in major enhancement of the production of IL-1beta in response to LPS. Am J Physiol Endocrinol Metab. 2006;290(3):E490-E493.

23. Simpson KL, Keelan JA, Mitchell MD. Labor-associated changes in interleukin-10 production and its regulation by immunomodulators in human choriodecidua. J Clin Endocrinol Metab. 1998;83(12):4332-4337.

24. Livak KJ, Schmittgen TD. Analysis of relative gene expression data using real-time quantitative PCR and the 2(-Delta Delta C(T)) Method. Methods. 2001;25(4):402-408.

25. Fortunato SJ, Menon R, Lombardi SJ. Amniochorion gelatinasegelatinase inhibitor imbalance in vitro: a possible infectious pathway to rupture. Obstet Gynecol. 2000;95(2):240-244.

26. Skinner SJ, Campos GA, Liggins GC. Collagen content of human amniotic membranes: effect of gestation length and premature rupture. Obstet Gynecol. 1981;57(4):487-489.
27. Pulukuri SM, Patibandla S, Patel J, Estes N, Rao JS. Epigenetic inactivation of the tissue inhibitor of metalloproteinase-2 (TIMP-2) gene in human prostate tumors. Oncogene. 2007;26(36):5229-5237.

28. Egger G, Liang G, Aparicio A, Jones PA. Epigenetics in human disease and prospects for epigenetic therapy. Nature. 2004;429(6990):457-463.

29. Terry SY, Vallis KA. Relationship between chromatin structure and sensitivity to molecularly targeted auger electron radiation therapy. Int J Radiat Oncol Biol Phys. 2012;83(4):1298-1305.

30. Nguyen CT, Weisenberger DJ, Velicescu M, et al. Histone H3-lysine 9 methylation is associated with aberrant gene silencing in cancer cells and is rapidly reversed by 5 -aza-2'-deoxycytidine. Cancer Res. 2002; 62(22):6456-6461.

31. Zheng Z, Li L, Liu X, et al. 5-Aza-2'-deoxycytidine reactivates gene expression via degradation of $\mathrm{pRb}$ pocket proteins. FASEB J. 2012;26(1):449-459.

32. Schmelz K, Sattler N, Wagner M, Lübbert M, Dörken B, Tamm I. Induction of gene expression by 5 -Aza-2'-deoxycytidine in acute myeloid leukemia (AML) and myelodysplastic syndrome (MDS) but not epithelial cells by DNA-methylation-dependent and -independent mechanisms. Leukemia. 2005;19(1):103-111.

33. Zhu WG, Dai Z, Ding H, et al. Increased expression of unmethylated CDKN2D by 5 -aza-2'-deoxycytidine in human lung cancer cells. Oncogene. 2001;20(53):7787-7796.

34. Chernov AV, Sounni NE, Remacle AG, Strongin AY. Epigenetic control of the invasion-promoting MT1-MMP/MMP-2/TIMP-2 axis in cancer cells. J Biol Chem. 2009;284(19):12727-12734.

35. Couillard J, Estève PO, Pradhan S, St-Pierre Y. 5-Aza-2'-deoxycytidine and interleukin-1 cooperate to regulate matrix metalloproteinase-3 gene expression. Int J Cancer. 2011;129(9):2083-2092.

36. Hernandez-Barrantes S, Bernardo M, Toth M, Fridman R. Regulation of membrane type-matrix metalloproteinases. Semin Cancer Biol. 2002;12(2):131-138.

37. Adams KM, Lucas J, Kapur RP, Stevens AM. LPS induces translocation of TLR4 in amniotic epithelium. Placenta. 2007;28(5-6):477-481.

38. Zampetaki A, Xiao Q, Zeng L, Hu Y, Xu Q. TLR4 expression in mouse embryonic stem cells and in stem cell-derived vascular cells is regulated by epigenetic modifications. Biochem Biophys Res Commun. 2006;347(1):89-99.

39. Takahashi K, Sugi Y, Hosono A, Kaminogawa S. Epigenetic regulation of TLR4 gene expression in intestinal epithelial cells for the maintenance of intestinal homeostasis. J Immunol. 2009;183(10):6522-6529.

40. Bernardo MM, Fridman R. TIMP-2 (tissue inhibitor of metalloproteinase-2) regulates MMP-2 (matrix metalloproteinase-2) activity in the extracellular environment after pro-MMP-2 activation by MT1 (membrane type 1)MMP. Biochem J. 2003;374(Pt 3):739-745.
Journal of Inflammation Research

\section{Publish your work in this journal}

The Journal of Inflammation Research is an international, peer-reviewed open-access journal that welcomes laboratory and clinical findings on the molecular basis, cell biology and pharmacology of inflammation including original research, reviews, symposium reports, hypothesis formation and commentaries on: acute/chronic inflammation; mediators of inflamma-

\section{Dovepress}

tion; cellular processes; molecular mechanisms; pharmacology and novel anti-inflammatory drugs; clinical conditions involving inflammation. The manuscript management system is completely online and includes a very quick and fair peer-review system. Visit http://www.dovepress.com/ testimonials.php to read real quotes from published authors. 